\title{
A blockchain-based cooperative modeling method for digital twin ontology model of the mechanical product
}

\author{
Menglei Zheng, and Ling Tian* \\ Department of Mechanical Engineering, Tsinghua University, Beijing, China
}

\begin{abstract}
With the rapid increase of multi-source heterogeneous dynamic data of mechanical products, the digital twin technology is considered to be an important method to realize the deep integration of product data and intelligent manufacturing. As a digital archive of the physical entity in entire life cycle, the mechanical product digital twin model is cross-phased and multi-domain. Therefore, safe and stable cooperative modeling has become a basic technical problem that needs to be solved urgently. In this paper, we proposed a blockchain-based collaborative modeling method for the digital twin ontology model of mechanical products. First, an authorization network was constructed among stakeholders. Then modeling processes of the digital twin were mapped to ontology operations and formatted through extensible markup language. Finally, consensuses were obtained based on practical byzantine fault tolerance. And a material modification process of a helicopter damper bearing was taken as an example to verify. The proposed method enables all participants to accurately obtain the latest state of the digital twin model, and has the advantages of tamper-proof, traceability, and decentralization.
\end{abstract}

Keywords: Digital twin, Blockchain, Collaborative modelling, Mechanical product.

\section{Introduction}

With the development of the new generation of information and communication technology, digitization and networking have made the acquisition, use, control and sharing of data faster and cheaper. Since the rapid increase of multi-source heterogeneous dynamic data throughout the life cycle of mechanical products has become an important feature of intelligent manufacturing, these data have broad application prospects in various stages of the product, such as design, manufacturing, operation and maintenance. As an important method for realizing the dynamic integration of product multi-source heterogeneous data and the deep integration of cyber-physical systems, digital twin technology has attracted increasing attention from scholars and the industry [1]. Digital twins are described as digital

\footnotetext{
* Corresponding author: tianling@tsinghua.edu.cn
} 
representations of real-world entities or systems [2]. As one of the most important applications of digital twin technology, product digital twin is defined as the full-element reconstruction and digital mapping of the working progress and status of product physical entity in the virtual space [3].

However, there are still many basic technical issues need to be solved for product digital twin, and collaborative modeling is one of them [4]. As a digital archive of product in the entire life cycle, the digital twin model has complex content with a long developing cycle, so this process is generally inseparable from cooperation. With the widespread application of information technology in the manufacturing industry, the effective interaction and indepth integration between the upstream and downstream stakeholders of the product can create value more efficiently, thereby promoting their participation in the sharing and interaction of information. However, there are still many challenges in this collaborative process, such as security, stability, and traceability. In this context, blockchain has a good application prospect in digital twin modeling due to its anti-tampering, traceability, uniqueness and some other characteristics [5].

This paper is oriented to the collaborative modeling of mechanical product digital twins. Based on the ontology method, a blockchain-based digital twin collaborative modeling method for mechanical products is proposed, which can provide a safe and efficient collaboration mechanism for stakeholders. Finally, an improvement process of a helicopter damper bearing is taken as an example to verify the proposed method.

\section{Analysis of the characteristics of mechanical product digital twin and related work}

In this section, the characteristics of mechanical product digital twin are analysed, and the related research on product digital twin ontology modeling and the integration of blockchain and digital twin technology is briefly introduced and analysed as the basis of the subsequent proposed methods.

\subsection{Analysis on heterogeneity of product digital twin and research on ontology modeling}

The product digital twin model, as a full-element mapping of the entity, contains a large number of multi-source heterogeneous data, such as drawings, geometry, material parameters, sensor measurement values, etc. In order to better express the multidisciplinary and multi-domain coupling and interaction of mechanical products, ontology as a general knowledge representation method, its application in digital twin modeling has become one of the current research directions. For example, Singh et al. [6] transformed domain knowledge into a minimal data structure through ontology, thereby mapping, querying and managing digital twin databases. Bao et al. [7] proposed a new concept of product information unit based on ontology, and studied the modeling method of multisource heterogeneous data. Combing the ontology method, a universal expression can be established for various features, and as the data architecture of the digital twin model, stores various types of data structuredly, as shown in Figure 1(a).

Furthermore, the evolution process of the mechanical product digital twin model can be managed based on the established ontology model. Combined with the model, operations of product information such as adding, deleting, and modifying can be mapped to the addition and deletion of corresponding nodes and edges in the ontology model, as shown in Figure 1(b). Based on this method, the modeling of mechanical product digital twin can be realized by operating the nodes and edges of its ontology model. 


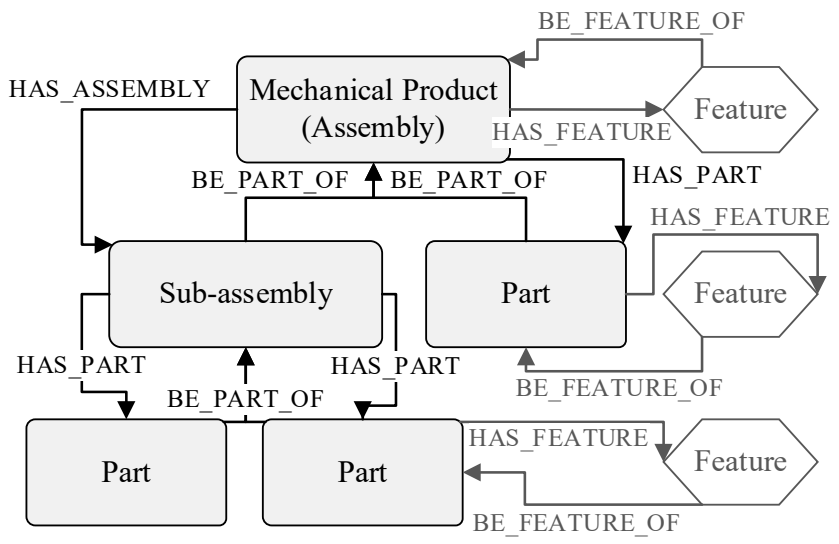

(a) Schematic diagram of product digital twin ontology model

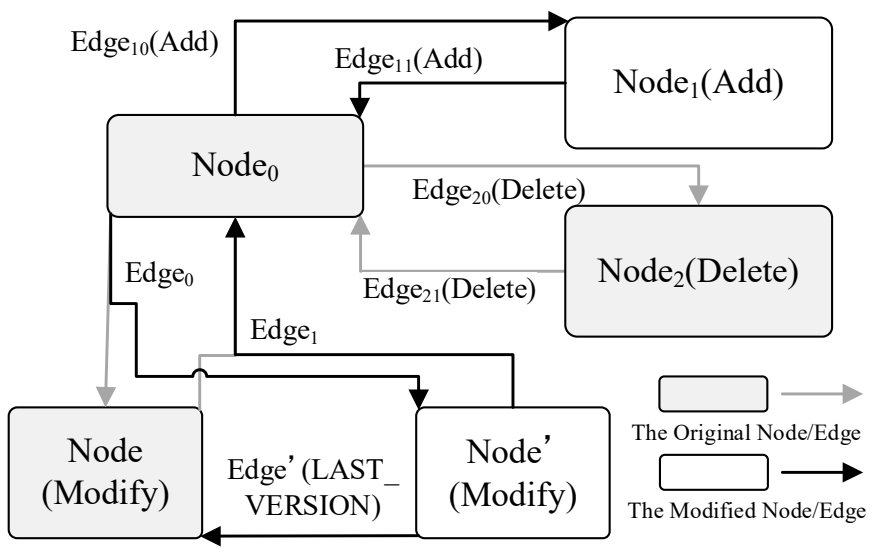

(b) Schematic diagram of the modification of product digital twin ontology model

Fig. 1. Ontology-based digital twin modeling method for mechanical products.

\subsection{Analysis on security of product digital twin and research on blockchain}

The cross-stage and multi-domain characteristics of the product digital twin determine that its modeling process is usually inseparable from cooperation. In terms of process, it often involves multiple departments or enterprises from upstream to downstream, such as designers, manufacturers, maintenance personnel and users; and in terms of content, experts from multiple fields are invited to collaborate and participate in modeling from design drawings, geometric models, process plans, operating data to simulation models. However, this process has many security challenges. Since the stakeholders involved may not fully trust each other, it is necessary to ensure that all data modification processes cannot be easily tampered with and are traceable. As the consensus of all participants on product information, the digital twin model needs to be unique and synchronized. Besides, although the development of a digital twin model is a win-win behaviour, it is often difficult to clarify the main benefits in the process. Therefore, partial decentralization is more suitable for solving the problem of sharing its ownership and modeling costs.

In this context, blockchain technology can give full play to its advantages. The blockchain is an ever-growing sequence of blocks that saves a complete list of transaction records and encrypts the data in conjunction with digital signatures [8]. The digital twin 
modeling method combined with the blockchain has been preliminary researched. Huang et al. [9] proposed a blockchain-based product digital twin data management method to identify and manage product configuration files and sensor data. Dietz et al. [10] proposed a secure digital twin data sharing framework based on distributed ledger technology, which combined with off-chain addresses to store structured and unstructured data. Because of the characteristics of tamper-proof, traceability, and decentralization of blockchain, it has good application prospects in product digital twin modeling.

\section{Methodology}

In response to the above requirements, this paper further combines the blockchain and ontology technology to propose a blockchain-based collaborative modeling method for the digital twin ontology model of mechanical products.

\subsection{Construction of an authorized network composed of involved stakeholders}

The blockchain systems are roughly categorized into public blockchain, private blockchain and consortium blockchain. Among them, all records in the public blockchain are visible to the public, with relatively high latency and low efficiency, while the private blockchain can only have nodes from a single organization. Therefore, for the digital twin model of mechanical products that requires the internal sharing of data from multiple stakeholders, the consortium blockchain is selected to build a partially decentralized system. Stakeholders must obtain authorization by registering their identity in the public key infrastructure, and then create their own nodes and establish connections to construct a distributed point-to-point authorization network. Each node has a corresponding address, public key, and private key.

Moreover, in order to achieve rapid acquisition of product digital twin information, each node will build a real-time ontology model locally based on the information in the blockchain. When joining the network for the first time, as the blockchain records the entire editing process of the product ontology model from its creation to the present, the node can develop the latest digital twin model by traversing the entire blockchain and reading the operations in turn.

\subsection{Request formatting and block content design for product digital twin ontology modeling}

After mapping the modeling process of the mechanical product digital twin to the iteration of the ontology model, all its modification processes can be classified into four operations: adding nodes, deleting nodes, adding edges, and deleting edges. In order to achieve reliable transmission of modeling information, the modification request is packaged through XML (EXtensible Markup Language), the format of which is shown in Figure 2(a). The nested content includes operation object, nonce, operation, attribute quantity and corresponding content.

A block consists of a block header and a block body. In addition to the genesis block, the block header includes the block version, timestamp, Merkle tree root hash, nonce, and hash of the parent block. The block header of the genesis block includes other information except the parent hash. The block body contains the request information formatted based on the XML method. A schematic diagram of the designed blockchain is shown in Figure 2(b). 


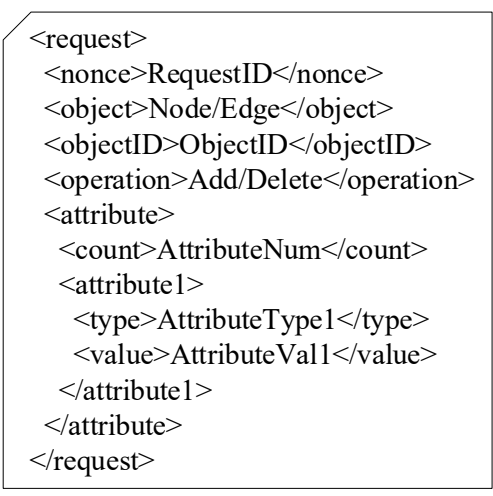

(a) Formatted Modification Request

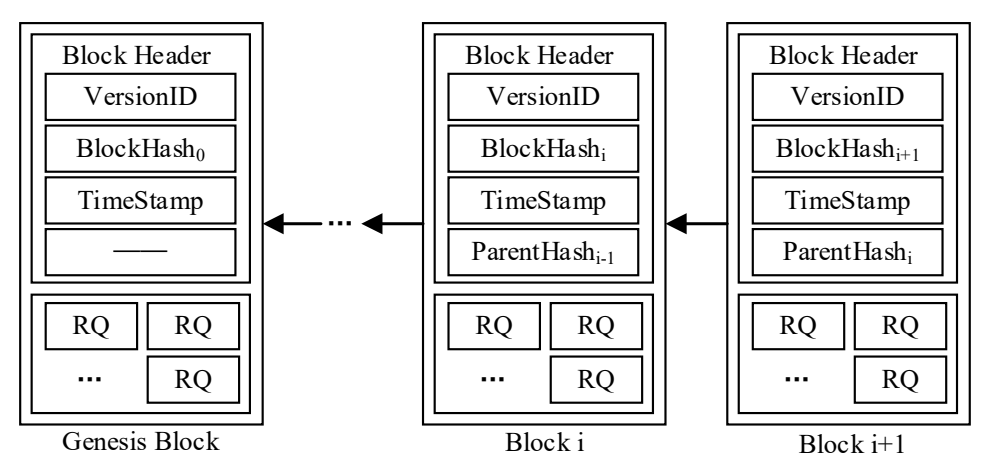

(b) Schematic diagram of the blockchain structure of product digital twin ontology modeling

Fig. 2. Request formatting and block design for product digital twin ontology modeling.

\subsection{PBFT-based product digital twin blockchain consensus and local model construction}

Considering that it is difficult to achieve complete synchronization in the actual network environment, the established digital twin ontology model blockchain nodes achieve consensus through the method based on the PBFT (Practical Byzantine Fault Tolerance) algorithm. One node in the network serves as the primary node, and the others are replica nodes. All nodes work under the same configuration information., and the consensus process is as follows:

- The client formats the request for adding or deleting the ontology and sends it to the primary node and replica nodes;

- The primary node adds a sequence number to the propose, calculates the pre-prepare message, and sends it to other replica nodes;

- When the replica node receives and verifies the pre-prepare message, it calculates the prepare message and broadcasts it; at the same time, all nodes collect prepare messages;

- When the node receives enough prepare messages, it calculates the commit message and broadcasts it, and adds the message to its local log;

- When any node receives enough commit messages, it sends a reply to the client, and the client confirms the completion.

If the primary node is unresponsive or is considered to be problematic by most other nodes, a view-change will occur, and the primary node will be replaced. According to the characteristics of the PBFT algorithm, the consensus process can be completed with less 
than one-third of the nodes failing. After completing a consensus, each node updates its local ontology model according to the latest received request information to keep the product digital twin model up to date.

\section{Case study}

The damper bearing is a component in the helicopter rotor system, which is composed of an inner ring, an outer ring, and a coating. This paper takes the modification of the coating material as an example, combined with a simplified model, to verify the proposed blockchain-based collaborative modeling method for the digital twin ontology model of mechanical products.

In the current network, there are four verified nodes: a designer, a manufacturer, a maintainer, and a user. According to the adversary model of the PBFT consensus, the maximum number of failed nodes is one. When the designer modifies the coating material, the operations that need to be performed on the ontology model include deleting the original material node and corresponding edge, and adding a new material node and corresponding edge, as shown in Figure 3. The designer node packages the request based on the proposed formatting method, and the obtained data is shown in Figure 4.

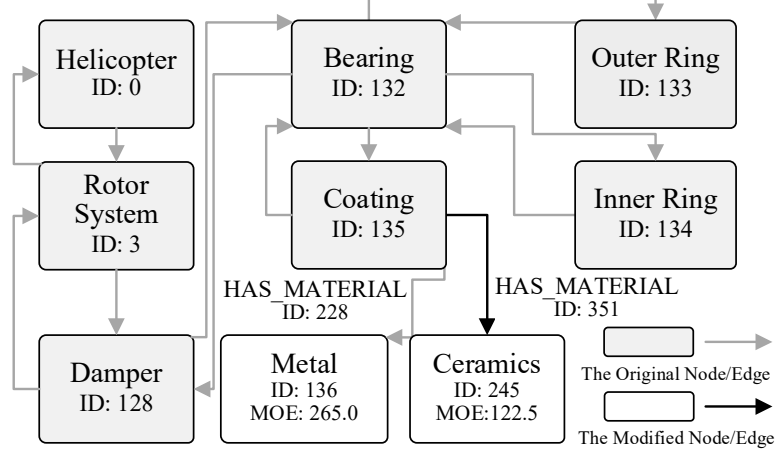

Fig. 3. Schematic diagram of coating material replacement process.
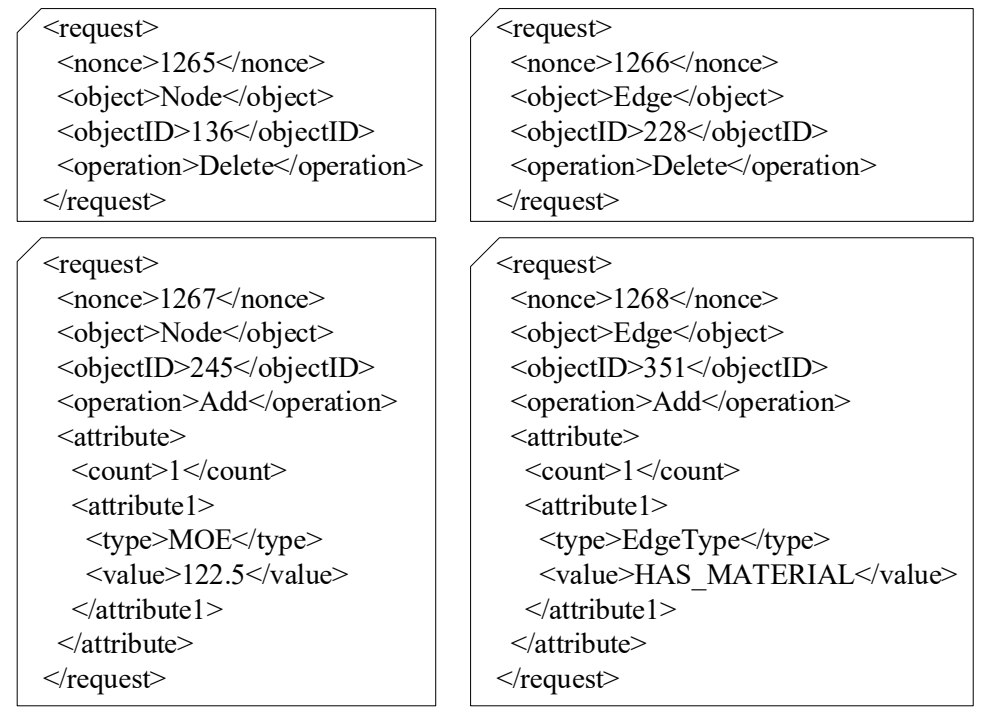

Fig. 4. Formatted operation request. 
As the client, the designer node triggers the consensus process after sending the request to other nodes:

- The primary node adds a sequence number to the propose, calculates the pre-prepare message, and sends it to three other replica nodes;

- When the replica node receives and verifies the pre-prepare message, it calculates the prepare message and broadcasts it; at the same time, all nodes collect prepare messages;

- When the node receives not less than three prepare messages, it calculates the commit message and broadcasts it, and adds the message to its local log;

- When any node receives not less than three commit messages, it sends a reply to the designer node, and the designer node confirms the completion.

\begin{tabular}{|c|c|c|c|c|c|}
\hline \multicolumn{2}{|c|}{ Block Header } & \multicolumn{2}{|c|}{ Block Header } & \multicolumn{2}{|c|}{ Block Header } \\
\hline \multicolumn{2}{|c|}{ Version: 1.0} & \multicolumn{2}{|c|}{ Version: 1.0} & \multicolumn{2}{|c|}{ Version: 1.0} \\
\hline \multicolumn{2}{|c|}{$\begin{array}{c}\text { 5e2f5d82288899cac85e9a } \\
\text { db557751d5e18190cc630b } \\
\text { d88ed34b92b4a751dda7 }\end{array}$} & \multicolumn{2}{|c|}{$\begin{array}{c}\text { 71d7a8b4dd3e7fb9830ec7 } \\
\text { ecaf9dd2c2da3f7e01663al } \\
\text { 67badb07e199d454e5c }\end{array}$} & \multicolumn{2}{|c|}{$\begin{array}{c}\text { 719edadec432b13a3b720c } \\
\text { d162958d9e3441f63bbd09 } \\
\text { b8682cdf3c33a2e541f9 }\end{array}$} \\
\hline \multicolumn{2}{|c|}{1622970603} & \multicolumn{2}{|c|}{1623142800} & \multicolumn{2}{|c|}{1623143400} \\
\hline & & \multicolumn{2}{|c|}{$\begin{array}{c}\text { cf676ea4e3622e 584c2def8 } \\
\text { 9c744e497f6c6d36f2cfdc } 1 \\
\text { 4c587c1c104cdlc235 }\end{array}$} & \multicolumn{2}{|c|}{$\begin{array}{c}\text { 71d7a8b4dd3e7fb9830ec7 } \\
\text { ecaf9dd2c2da3f7e01663al } \\
\text { 67badb07e 199d454e5c }\end{array}$} \\
\hline RQ & RQ & RQ & RQ & RQ1 & RQ2 \\
\hline$\cdots$ & RQ & $\cdots$ & RQ & RQ3 & RQ4 \\
\hline
\end{tabular}

Fig. 5. Schematic diagram of the blockchain after completing the update of the coating material.

After the consensus is completed, the latest state of the blockchain is shown in Figure 5. Each node uses the received request information to delete the original material node and corresponding edge, add a new material node and corresponding edge, and complete the collaborative modeling process of the product digital twin model on its own local ontology model.

\section{Conclusion}

Digital twin is an important method to deeply integrate product data and realize intelligent manufacturing, and collaborative modeling is one of the basic problems to be solved urgently. The blockchain-based collaborative modeling method for digital twin ontology model of mechanical products proposed in this paper, constructs an authorization network composed of stakeholders, uses XML to format the modification requests of the ontology model mapped from the digital twin, achieves consensus with PBFT method, and continuously develops new blocks and updates the twin model at each node. The proposed collaborative modeling method enables all participants to accurately obtain the latest state of the digital twin model, and has the advantages of tamper-proof, traceability, and decentralization, provides a feasible technical foundation for the product digital twin.

This work was supported by the National Key Research and Development Project of China [grant number 2018YFB1700604, 2020YFB1709100]; the Beijing Municipal Natural Science Foundation [grant number 3182012]; and the Tsinghua University Initiative Scientific Research Program [grant number 2018Z05JZY006]. 


\section{References}

1. Lim K Y H, Zheng P and Chen C H 2020 A state-of-the-art survey of Digital Twin: techniques, engineering product lifecycle management and business innovation perspectives. Journal of Intelligent Manufacturing, 31(6) pp 1313-1337.

2. Haag S and Anderl R 2018 Digital twin Proof of concept. Manufacturing Letters, 15 pp 64-66.

3. Zhuang C, Liu J, Xiong H, Ding X, Liu S and Weng G 2017 Connotation, architecture and trends of product digital twin. Computer Integrated Manufacturing Systems, CIMS, 23(4) pp 753-768.

4. Tao F and Qi Q 2019 Make more digital twins. Nature, 573(7775) pp 490-491.

5. Yaqoob I, Salah K, Uddin M, Jayaraman R, Omar M and Imran M 2020 Blockchain for Digital Twins: Recent Advances and Future Research Challenges. IEEE Network, 34(5) pp 290-298.

6. Singh S, Shehab E, Higgins N, Fowler K, Reynolds D, Erkoyuncu J A and Gadd P 2020 Data management for developing digital twin ontology model. Proceedings of the Institution of Mechanical Engineers, Part B: Journal of Engineering Manufacture.

7. Bao Q, Zhao G, Yu Y and Dai S 2021 Product Information Units Modeling Oriented to Digital Twin. IOP Conference Series: Earth and Environmental Science 2020 2nd International Conference on Resources and Environmental Research, ICRER 2020.

8. Zheng Z, Xie S, Dai H, Chen X and Wang H 2017 An Overview of Blockchain Technology: Architecture, Consensus, and Future Trends. Proceedings - 2017 IEEE 6th International Congress on Big Data.

9. Huang S, Wang G, Yan Y and Fang X 2020 Blockchain-based data management for digital twin of product. Journal of Manufacturing Systems, 54 pp 361-371.

10. Dietz M, Putz B and Pernul G 2019 A distributed ledger approach to digital twin secure data sharing. Lecture Notes in Computer Science. 